https://doi.org/10.32735/S0718-2201202100052893

243-260

\title{
CRÍTICA AL MULTICULTURALISMO LIBERAL, HACIA UN MULTICULTURALISMO CRÍTICO
}

A critique of liberal multiculturalism, towards a critical multiculturalism.

\author{
JOSÉ MANUEL FERNÁNDEZ RUIZ \\ Universidad Alberto Hurtado (Chile) \\ jmfernandez@uaburtado.cl
}

Resumen

En este ensayo desarrollo una crítica al modelo de justicia multicultural de Will Kymlicka, con el propósito de establecer las bases para el desarrollo de un multiculturalismo crítico. El argumento se ordena de la siguiente forma. En la primera sección analizo la teoría de los derechos especiales de Kymlicka e identifico el valor liberal central al que estos responden, la autonomía individual. En la segunda sección examino el fundamento igualitarista de la suerte de su teoría de los derechos especiales. En la tercera sección elaboro una crítica general a la justicia multicultural liberal desarrollando un argumento para politizar los reclamos de los pueblos indígenas para el acceso a su cultura. Argumento que el trato justo de las minorías indígenas requiere reconocer su autodeterminación política.

Palabras clave: Multiculturalismo crítico; igualitarismo de la suerte; teoría crítica; pueblos indígenas.

\section{Abstract}

In this essay I develop a critique of Will Kymlicka's model of multicultural justice, with the purpose of laying down the foundations for a critical multiculturalism. The argument is ordered in the following way. In the first section I analyse Kymlicka's theory of special rights and identify the central liberal value to which they respond: individual autonomy. In the second section I examine the luck egalitarian foundations of Kymlicka's theory of special rights. In the third section, I elaborate a general critique to liberal multicultural justice, developing an argument to politicize the claims of indigenous peoples to access to their culture. Basically I argue that the just treatment of indigenous minorities requires recognizing their political self-determination.

Key words: Critical multiculturalism; luck egalitarianism; critical theory; indigenous peoples.

\section{INTRODUCCIÓN}

Este es un trabajo de filosofía política, en específico respecto del multiculturalismo y un intento de politizar y radicalizar una interpretación de sus fundamentos que parece estar siendo socavado al menos en ciertas áreas de intervención del Estado y que al realzarlo, pretende ofrecer un sustento más robusto para ciertas demandas contra el Estado efectuadas por los pueblos indígenas, en particular, la demanda por diversas formas de autonomía colectiva. En esta tarea de filosofía política, es decir, de investigación respecto de los fundamentos y justificaciones del ejercicio del poder 
político (Miller, 2006, p. 815), utilizaré el término "multicultural" en contraste al término "interculturalidad". La razón de esta elección es que el fundamento filosófico que propongo para una filosofía política multicultural capaz de legitimar la actividad estatal está destinado a especificar las condiciones que el Estado debería cumplir para considerar que ha tratado con "justicia" a las minorías indígenas.

Esto debo subrayarlo; el objetivo del presente trabajo reside en identificar el fundamento del tratamiento especial de las minorías indígenas, y en principio, no el de otras minorías. Es decir, la propuesta identifica un principio de justicia política que el Estado se encuentra obligado a respetar en su trato con los pueblos indígenas. No respetar este tipo de tratamiento, no cumplir la condición, si se trata de otras minorías, no implica una vulneración de los principios de justicia política que legitiman el actuar del Estado. Sin embargo, si no se cumple dicha condición tratándose de los pueblos indígenas, el trato debe considerarse injusto, y en principio, ilegítimo. Este principio de justicia consiste sobre todo en asegurar el acceso a los pueblos indígenas a "su cultura", entendido como un acceso de carácter sustantivo. Esta elaboración particular de una filosofía política del multiculturalismo contrasta con una que podría justificarse en lo "intercultural". En el debate moderno acerca del multiculturalismo, en particular en el ámbito europeo, se ha insistido en la superioridad de una filosofía política intercultural por sobre una multicultural. Sin embargo, tal enfoque es perjudicial cuando se trata del reconocimiento de la autonomía colectiva de pueblos indígenas. En efecto, el "interculturalismo" aparece como retórica en un espacio político que considera el fracaso del "multiculturalismo" por haber tenido como efecto un socavamiento de ciertos derechos individuales y contribuido a la segregación supuestamente por su enfoque enfático en los derechos de las colectividades (Kymlicka, 2014, p. 160), como los pueblos indígenas.

La razón por la que la fundamentación de las demandas indígenas deben ser carácter multicultural por sobre aquellas de carácter intercultural, es que un énfasis exclusivo en la interculturalidad es una forma de justificar los arreglos sociales básicos en lo que la discusión acerca de la autonomía colectiva de los grupos indígenas queda por definición excluida. Una defensa de la "interculturalidad" apela a las microinteracciones, a los contactos entre individuos pertenecientes a diferentes culturas en un mundo de superdiversidad. Una política intercultural si bien avanza a este micronivel de interacciones que no son de manera habitual el objeto de atención del multiculturalismo (Kymlicka, 2014, p. 160); no obstante, debe dejar de lado las relaciones entre el Estado y los pueblos originarios porque esta es una relación "entre naciones" (Bouchard, 2011, p. 439; Taylor, 2012, p. 416). Este tipo de interacciones son sobre todo las que una filosofía política del multiculturalismo crítico pretende desarrollar. Una filosofía política de la interculturalidad debería proceder en la identificación de los términos que constituyen un diálogo apropiado entre diversas culturas, pero no enfatiza aquello que es distintivo de una cultura y por esta razón no 
considera de principio los reclamos a la autodeterminación de grupos que han sido objeto de injusticias históricas, como los pueblos indígenas.

\section{LA TEORÍA DE LOS DERECHOS DE LAS MINORÍAS DE WILL KYMLICKA}

Partir examinando la teoría de los derechos de las minorías culturales desarrollada por Will Kymlicka es importante porque él la ha desarrollado teniendo a la vista ciertas descripciones de la sociedad canadiense que son comunes al modelo de estado liberal europeo. Es decir, estas descripciones son comunes a muchos Estados, como el chileno, que han tomado el modelo de estado liberal europeo para la organización política de la sociedad. Por esta razón, cuando Kymlicka hace manifiesto los compromisos valóricos de este modelo de Estado en su relación con las minorías culturales, pone de relieve consideraciones importantes respecto de su trato justo que son en general aplicables a todos los Estados que siguen dicho modelo, y que no han sido de manera suficiente reconocidas en el debate público de los derechos de las minorías. Kymlicka ha logrado subrayar las bases sobre la que se asientan ciertas concepciones morales y políticas que legitiman al Estado, así como el compromiso con ciertos valores fundamentales, como la autonomía individual y la diversidad cultural, y cómo ambos se encuentran de forma estrecha relacionados. En ello reside la importancia de su obra filosófica y política: en haber hecho explícito los fundamentos para la protección de las minorías y que estos subyacen a los compromisos valóricos del Estado liberal.

Aunque comparto muchas de las ideas desarrolladas por Kymlicka acerca de los derechos de las minorías, creo que el énfasis en los derechos individuales ha resultado en un enfoque teórico despolitizado, perdiendo, en definitiva, su potencial crítico y emancipatorio. De hecho, esta es una de las motivaciones de este trabajo, enfatizar una alternativa de politización de los reclamos de las minorías culturales, en especial de los pueblos indígenas. El multiculturalismo de Kymlicka parte reconociendo un punto básico: gran parte de las sociedades liberales contemporáneas son multiculturales. Kymlicka toma esta descripción como verdadera y explica cuáles son las dos fuentes principales del pluralismo cultural. Por un lado, el pluralismo cultural se deriva de la absorción por parte de ciertos Estados de naciones autodeterminadas preexistentes (Kymlicka, 1995, p. 11). Kymlicka denomina a estas naciones autodeterminadas preexistentes minorías nacionales. Por otro lado, el pluralismo cultural proviene de flujos migratorios de un país a otro, grupos sobre todo de inmigrantes que Kymlicka denomina grupos étnicos (p. 14). Tomando en consideración estas dos fuentes del multiculturalismo define qué es posible entender por cultura. Vale la pena señalar que esta no es la única definición de cultura que formula. Con posterioridad, en su libro Ciudadanía multicultural, introduce otra noción de cultura que denomina "cultura societal", que no está relacionada, al menos en un sentido obvio, con su definición anterior de cultura y que 
ha sido objeto de varios malentendidos. La relación entre las dos definiciones se especificará a continuación.

Por ahora es necesario centrarse en la primera definición de cultura. Según Kymlicka, la primera definición de cultura tiene por objeto establecer un criterio para poder caracterizar a ciertos Estados como multiculturales. Si la noción de cultura es demasiado amplia, cualquier país podría considerarse multicultural. De hecho, una noción amplia de cultura puede aplicarse a la cultura legal, burocrática, o política, considerando diversas agregaciones de individuos. Por tanto, bajo esta descripción, cualquier Estado con este tipo de agregaciones de individuos se contaría como multicultural, conclusión que Kymlicka rechaza porque volvería al multiculturalismo, como concepción de justicia política, superflua. Kymlicka (1995) formula una concepción bastante estricta de cultura:

como sinónimo de "nación" o "pueblo" esto es, una comunidad intergeneracional, más o menos institucionalmente completa, que ocupa un determinado territorio o tierra natal, y que comparte un lenguaje e historia distintiva. Y un Estado es multicultural si sus miembros o pertenecen a diferentes naciones (Estado multinacional), o han emigrado de diferentes naciones (Estado poli-étnico), y si este hecho es un importante aspecto de su identidad y vida política (p. 18, trad. del autor).

Equipado con esta primera definición de cultura, la propuesta de Kymlicka no solo es capaz de distinguir entre países multiculturales y no multiculturales, sino que también puede distinguir entre grupos que enfrentan la asimilación cultural de maneras diferentes. En efecto, su propuesta distingue entre minorías nacionales y grupos étnicos, diferencia que en la teoría de Kymlicka es fundamental para fijar cuál es el grado apropiado de respeto por las minorías al que el Estado liberal se encuentra obligado. Bajo la premisa que los Estados multiculturales tienen dos fuentes de pluralismo cultural, Kymlicka, de manera consistente, caracteriza a estas dos clases de minorías culturales como si persiguieran objetivos distintos. Las minorías nacionales, grupo entre los que se agrupa tanto el caso de Quebec, como Escocia y Puerto Rico, y la situación de los pueblos indígenas, tienen como objetivo mantener un carácter distintivo; persiguen ser vistos y autocomprenderse como una nación distinta de aquella más amplia en la que existen. Quebec está situado en Canadá, Escocia en el Reino Unido, Puerto Rico en Estados Unidos, y los diversos pueblos indígenas, como el Mapuche y el Rapa-Nui, en Chile. De acuerdo con Kymlicka las minorías nacionales tienen por objetivo, y de hecho ocupan, parte del territorio en el que otros Estados ejercen ahora su soberanía, y no solo consideran que tienen derecho a un territorio en particular, sino que también merecen ser tratados como naciones que se autodeterminan como naciones independientes.

Los grupos étnicos, en particular grupos inmigrantes, por el contrario, Kymlicka los caracteriza de manera diferente. De acuerdo con Kymlicka los grupos étnicos o grupos 
de inmigrantes tienen una autocomprensión diversa. Estos grupos no pretenden ser vistos ni comprendidos como una nación distinta, ni pretenden ocupar un determinado espacio del territorio del Estado de manera exclusiva. Su particularidad deriva de sus vínculos familiares y asociaciones especiales (p.14). La caracterización de ambos grupos como grupos que persiguen finalidades distintas no es superflua, porque esta caracterización determina que estos sean acreedores de diverso tipo de tratamiento. Determina, entonces, que sean concebidos como titulares de ciertos derechos y ciertas prerrogativas diferentes. Ahora bien, de que Kymlicka establezca una caracterización de diversos grupos minoritarios que persiguen acceso a sus prácticas y valores culturales de manera diferenciada, no se sigue que el fundamento que justifica un tratamiento especial sea diferente. De hecho, el tratamiento especial de ambos, minorías nacionales y grupos étnicos, en la teoría de Kymlicka, tiene el mismo fundamento. Este fundamento Kymlicka lo desarrolla por la descripción del Estado liberal que pretende proteger, promover, y desarrollar el valor de la autonomía individual (p. 75). En otras palabras, la justificación de derechos especiales para estas minorías, para que puedan acceder a sus prácticas y valores en posición de igualdad con relación a la mayoría, reside en un valor fundamental; el valor de la autonomía de los individuos para la elección apropiada de sus planes de vida.

La pregunta es cuál sería la relación entre estos derechos especiales para las minorías y el valor de la autonomía individual, de manera tal que se justifique un tratamiento especial que las favorezca. Es aquí donde Kymlicka introduce su segunda definición de cultura, la "cultural societal". Por cultura societal Kymlicka entiende

una cultura que proporciona a sus miembros formas de vida significativas en toda la gama de actividades humanas, incluidas la vida social, educativa, religiosa, recreativa y económica, que abarca esferas tanto públicas como privadas ... Tales 'culturas societales' no siempre existieron, y su creación está íntimamente vinculada con el proceso de modernización (p. 76).

Hay una razón para considerar que estas culturas sociales surgen solo en la modernidad y que está conectada con el valor que las culturas, en el primer sentido, tienen hoy para la autonomía individual ${ }^{1}$. Para aclarar en mayor medida las implicancias de estas dos concepciones de cultura introduzco una terminología para diferenciarlas. La primera definición de cultura la nombraré $\mathrm{CU}_{1}$, y la segunda la nombraré $\mathrm{CU}_{2}$. Kymlicka considera que, en las condiciones de la modernidad, para poder mantener y hacer florecer

\footnotetext{
${ }^{1}$ Carens (2000, Capítulo 3) ha argumentado, de forma errónea en mi opinión, que para Kymlicka, las culturas de los pueblos indígenas surgieron en la modernidad, ya que las culturas sociales no existían antes. Del mismo modo, Parekh (2000, p. 101) afirma, de manera errónea, que Kymlicka utiliza la cultura social y la cultura de forma indistinta como si fueran sinónimos. Sin embargo, esto es incorrecto porque la noción de culturas societales busca identificar las condiciones institucionales en donde los contextos culturales serían significativos en los tiempos modernos, mientras que la noción de cultura se refiere a los contextos para una elección significativa.
} 
$\mathrm{CU}_{1}$ esta debería poder transformarse en $\mathrm{CU}_{2}$ (Kymlicka, 1995, p. 80). Solo si esta condición se cumple, entonces $\mathrm{CU}_{1}$ puede tener valor, no solo desde un punto de vista liberal, sino como contexto para la elección autónoma. $\mathrm{CU}_{2}$, en la concepción de Kymlicka, es una especie de criterio para determinar cuándo $\mathrm{CU}_{1}$ tiene valor: solo cuando $\mathrm{CU}_{1}$ tiene las propiedades de $\mathrm{CU}_{2}$ puede perdurar bajo las condiciones de la modernidad y ser en consecuencia un contexto significativo que el Estado liberal debe resguardar. Hay que subrayar el papel que cumple $\mathrm{CU}_{2}$ para $\mathrm{CU}_{1}$ : las culturas minoritarias solo pueden sobrevivir en las condiciones de la modernidad si son culturas societales. Relacionadas de esta manera el lector ya puede anticiparse a la conclusión a la que Kymlicka quiere llegar. Esto es, que el valor básico que promueven las culturas societales y que revela su naturaleza instrumental es el valor liberal de la autonomía individual:

En pocas palabras, la libertad implica el poder elegir entre varias opciones, y nuestra cultura societal no solo proporciona estas opciones, sino que también las hace significativas para nosotros.

Las personas toman decisiones sobre las prácticas sociales que los rodean, en función de sus creencias sobre el valor de estas prácticas... Y tener una creencia sobre el valor de una práctica es, en primer lugar, una cuestión de entender los significados que nuestra cultura le atribuye (Kymlicka, 1995, p. 83, trad. del autor).

El argumento de Kymlicka persigue mostrar la base sobre la que se justifican los derechos especiales para las minorías. En sentido negativo: no hay base para los derechos especiales, en las condiciones de la modernidad, si $\mathrm{CU}_{1}$ no constituye un entorno capaz de dar sentido a las acciones individuales y, por tanto, fomentar la autonomía individual. $\mathrm{CU}_{1}$ debe poder convertirse en $\mathrm{CU}_{2}$ para constituir un contexto valioso, porque solo es valioso si fomenta la autonomía individual. La autonomía individual aparece como el valor central que persigue proteger y promover el multiculturalismo, lo que es naturalmente fundamental para determinar la valoración de esta doctrina al acceso de las minorías a su cultura. En efecto, para Kymlicka, y lo que describe creo de forma acertada la estructura de la política liberal del multiculturalismo, $\mathrm{CU}_{1}$ no es un bien en sí mismo, pero solo en la medida en que proporciona a las personas "acceso a una gama de opciones significativas" (p. 83). Para el multiculturalismo liberal, solo si CU $\mathrm{CU}_{1}$ es necesario para el desarrollo o fomento de la autonomía individual, entonces se justifica la existencia de medidas estatales para la protección de $\mathrm{CU}_{1}$ de modo que emerja como $\mathrm{CU}_{2}$. Kymlicka no busca ofrecer una justificación incondicionada de los derechos de las minorías que deba ser institucionalizada e implementada por el Estado, porque la concesión de las medidas especiales está supeditada a que estas medidas resguarden la autonomía individual. En otras palabras, la protección del acceso de las minorías a su propia cultura es instrumental. Debido a que el fin último de los derechos multiculturales es resguardar y proteger la autonomía individual, las minorías son valiosas porque estas constituyen un 
medio para que los individuos tomen elecciones autónomas (p. 84). Esto lo que en definitiva significa "acomodar las diferencias culturales". Veamos ahora un poco más de cerca la "forma liberal" de esta filosofía política multicultural.

Una vez zanjada la cuestión relativa a la identificación del valor central sobre la que se erige la política multicultural liberal, Kymlicka desarrolla una tipología específica de derechos que proteja de manera suficiente el acceso de las minorías a sus culturas y que les permitan a los individuos adaptarse a las demandas de la sociedad en la que se encuentran (p. 26). De acuerdo con Kymlicka hay tres tipos de derechos especiales que podrían quizá establecerse para las minorías: (1) derechos de autodeterminación o autogobierno, (2) derechos poliétnicos y (3) derechos especiales de representación (p. 27). Estos tres tipos de derechos básicos no se distribuyen por igual para cada tipo de minoría. Recordemos que según Kymlicka hay dos tipos de origen que hacen a un país multicultural y que se corresponden con dos tipos diferentes de minorías. A base de la caracterización de estas minorías Kymlicka considera que solo las minorías nacionales pueden ser beneficiados con derechos del tipo (1). Ejemplos son los derechos que existen para ciertas minorías nacionales como Quebec, Escocia y Puerto Rico, en las que bajo ciertos arreglos de carácter federal o semifederal, se entrega por parte de Canadá, Inglaterra y Estados Unidos, respectivamente, ciertas facultades legislativas respecto de ciertas materias. También constituyen formas del tipo (1) las comunidades indígenas que tienen ciertas competencias jurisdiccionales y administrativas, como el caso de los Navajo en Estados Unidos, los Maori en Nueva Zelanda, y los Inuit en Canadá.

Para Kymlicka estos derechos de auto-autogobierno en principio solo pueden ser concedidos a las minorías nacionales, lo que se justifica por el tipo de minorías que son. En consecuencia, los grupos étnicos no pueden verse beneficiados por derechos del tipo (1). Sin embargo, tanto las minorías nacionales como los grupos étnicos pueden verse beneficiados con derechos del tipo (2): derechos a recibir fondos públicos para financiar ciertas prácticas culturales y ciertas normas que los eximen de cumplir con ciertas cargas públicas. Esta sería a grandes rasgos la situación de los pueblos indígenas en Chile. Ni los Rapa-Nui ni Mapuche, ni ningún otro pueblo indígena, ha sido beneficiado con derechos del tipo (1), lo que indica desde ya, lo lejos que nuestro país se encuentra de regulaciones más enfáticas en la protección de los derechos de los pueblos indígenas. Es importante destacar, en todo caso, que el establecimiento de derechos restringidos de autogobierno a los pueblos indígenas de Canadá, Nueva Zelandia y Estados Unidos, no ha transformado a estos países en iliberales ni mucho menos. Se trata, en efecto, también de políticas liberales del multiculturalismo, solo que son políticas mucho más profundas y enfáticas en la protección de los derechos de estas minorías. Derechos del tipo (3) en principio solo parecen poder beneficiar a minorías nacionales, porque se trata de ciertos derechos que refuerzan la representación de ciertas minorías tradicionalmente infrarrepresentadas en los poderes públicos, como el legislativo y el ejecutivo. De más está decirlo que en Chile 
tampoco hoy los pueblos indígenas han sido beneficiados por este tipo de derechos (solo en el contexto excepcional de hoy parece posible que los pueblos originarios puedan acceder a medidas especiales de representación política).

Sea como sea que describamos la política multicultural chilena, lo cierto es que incluso en las alternativas más robustas de protección del acceso a prácticas culturales como el enfoque liberal en los países mencionados, previa la propuesta filosófica de Kymlicka, sigue estando presente bajo la superficie. Tomando esta orientación fundamental del resguardo de la autonomía individual, Kymlicka propone distinguir en cuanto a los efectos de su tipología de derechos, entre efectos que no se justifican bajo un paradigma liberal y efectos que sí se justifican. La distinción clave a este respecto es la distinción que articula entre restricciones internas y protecciones externas. Cuando los derechos especiales operan como restricciones internas (Kymlicka, 1995, p. 36), estas autorizan a las minorías a imponer a sus propios miembros diversas formas de control de la disidencia. Cuando los derechos especiales operan como protecciones externas (p. 36), estas implican protecciones contra las influencias originadas desde el exterior del grupo. Mientras que las restricciones internas quizá podrían autorizar la violación de derechos liberales básicos, las protecciones externas están destinadas a prevenir interferencias indebidas en el ejercicio de la cultura del grupo. En otras palabras, los derechos especiales que propone Kymlicka no se justifican si estas tienen como consecuencia restricciones internas. Sin embargo, sí son permisibles los derechos especiales cuando estos tienen como consecuencia protecciones externas. En suma, la propuesta de derechos especiales y que es a grandes rasgos la que adoptan los diversos países ya mencionados, encuentra su límite en la violación de derechos liberales considerados básicos. La justificación de dicho límite aparece, desde luego, como una forma de proteger la autonomía individual de las personas. El modelo aparece entonces como un modelo liberal de multiculturalismo.

\section{EL IGUALITARISMO DE LA SUERTE Y LA DESIGUALDAD EN EL ACCESO A CU 2}

En la sección precedente identifiqué el valor central que se protege con la estructura de derechos especiales ofrecida por Kymlicka, sin embargo, todavía es necesario una explicación de por qué estos derechos son indispensables. Mientras que en la sección anterior traté de identificar el valor central al que responde la política liberal multiculturalista, esta sección tiene por propósito examinar las razones que explican que el contexto que hace necesario estos derechos especiales. En efecto, pareciera que el hilo conductor de este ensayo no ha sido lineal, porque aceptado que el valor central del modelo de Kymlicka es la autonomía individual, uno podría considerar que existen otras medidas alternativas disponibles para el Estado para fomentar y proteger a las minorías. La pregunta es qué es lo que justifica los derechos especiales para el acceso igualitario a la cultura. Aquí 
aparece una segunda línea argumentativa seguida por Kymlicka: la fundamentación igualitarista de los derechos especiales. En concreto, el argumento de Kymlicka consiste en haber identificado una violación del principio de igual trato al que está comprometido el estado liberal. En otras palabras, la existencia de un trato desigual es lo que según Kymlicka haría necesario la existencia de derechos especiales. Estos derechos:

compensan las circunstancias desiguales que colocan a los miembros de las minorías culturales en desventaja sistémica en el mercado de la cultura, independientemente de sus elecciones personales de vida. Esta es una de las tantas áreas en las cuales la verdadera igualdad requiere no un trato idéntico, sino más bien un trato diferencial en orden a acomodar necesidades diferenciales (Kymlicka, 1995, p. 113, trad. del autor).

Desde el punto de vista de la justicia igualitaria, Kymlicka afirma que nacer en una cultura determinada, inspirada en valores específicos y sujeta a patrones de comportamiento distintivos, es una circunstancia no elegida. Caracterizar la cultura en la que nacemos como una circunstancia no elegida es relevante, porque esto significa que mientras la mayoría accede a una $\mathrm{CU}_{2}$ segura de forma gratuita, ya que es la cultura de la mayoría la que domina un territorio determinado como una ciudad, para las minorías el acceso es costoso. Los miembros de las culturas minoritarias, tanto las minorías nacionales como los grupos étnicos, no pueden acceder de manera gratuita a sus $\mathrm{CU}_{2}$. Ellas y ellos deben "pagar el costo" de no vivir en un entorno en el que su cultura es dominante, y por tanto deben pagar para acceder a ella. El término "costo" es entendido aquí en sentido amplio, no reducible a una significación económica. Por ejemplo, si un extranjero desea colocar a sus hijas en un colegio en Chile, pero proviene de un país donde no se habla español, tanto los hijos como los padres deberán pagar un costo. Las hijas un costo de "adaptación" y los padres el costo, por ejemplo, de contratar traductores o profesores para aprender el idioma. En la medida que podamos entender que la decisión de emigrar a Chile pueda entenderse como "voluntaria", no parece implausible considerar que el costo deba soportarlo quien decide residir aquí. No sucede lo mismo cuando tal decisión no es voluntaria, como sucede de manera habitual con las minorías nacionales y los refugiados. En estos casos, cuando no parece cumplirse con las exigencias mínimas de justicia igualitaria, sin derechos especiales las minorías se encontrarían en una situación desventajosa vis a vis a la mayoría. Esta situación desventajosa es considerada una violación de los principios liberales de justicia, y por tanto, para evitar un trato desigual, se deben establecer derechos especiales para alcanzar un tratamiento justo.

En esta justificación de los derechos de las minorías basada en la desigualdad del acceso a la cultura, Kymlicka recurre a la premisa tradicional de la doctrina liberal que establece que, en principio, las personas deben hacerse responsable de sus decisiones. En consecuencia, si X es responsable de su decisión, X es responsable de soportar los costos 
que se generan como efecto de su decisión ${ }^{2}$. Este énfasis en la responsabilidad individual como criterio para determinar si una persona debe recibir ayuda del Estado para soportar los costos de sus decisiones, está muy influenciado por la teoría liberal de la justicia de John Rawls. Como muestra Kasper Lippert-Rasmussen, Kymlicka proporciona una justificación de los derechos de las minorías de acuerdo con la noción de estructura básica Rawlsiana (Lippert-Rasmussen, 2011, p. 177). Para que las minorías puedan tener un contexto cultural seguro de elección estas necesitan mejorar su situación actual. En términos de las definiciones de cultura ofrecida por Kymlicka, $\mathrm{CU}_{1}$ requiere transformarse en $\mathrm{CU}_{2}$. El acceso a un contexto cultural es un bien que cualquier individuo racional desearía independientemente de la vida que quiere vivir. Sin embargo, mientras que la mayoría puede acceder a su cultura de forma gratuita, las minorías deben pagar por ello. Como las desigualdades que sufren las minorías en el acceso a sus culturas no se deben a sus elecciones, porque nacer en una cultura, como hemos visto, es una circunstancia no elegida, entonces estas deben ser compensadas, y la forma en que ello se produce es requiriendo del Estado algunos de los derechos positivos especiales de las formas (1) a (3).

La perspectiva filosófica utilizada por Kymlicka para evaluar si las elecciones pueden ser compensadas se denomina en la filosofía política igualitarismo de la suerte. El igualitarismo es aquella doctrina política que considera que todos los seres humanos son iguales, y que por tanto deben ser considerados y tratados como iguales (Arneson, 2013, en línea). En particular en el área de la justicia política, el igualitarismo es una doctrina política de la justicia que refiere a la correcta forma de distribución de recursos u otros factores moralmente relevantes entre las personas (Hirose, 2015, p. 2). Desde luego, no toda diferencia de distribuciones es moralmente relevante, sino que en principio solo aquellas que pueden hacer alguna diferencia en el bienestar individual. El igualitarismo se denomina de la "suerte" cuando tiene por objetivo contrarrestar los efectos de la "mala suerte" en la vida de las personas (Knight, 2013, pp. 924-925). No toda mala suerte, eso sí, es considerada apta para ser contrarrestada. En efecto, como Ronald Dworkin (2013) afirma, solo los efectos de la mala suerte que no se siguen de las decisiones de los individuos es aquella que debe ser compensada (Hirose, 2015, p. 2). Aplicada al acceso a la cultura, se arriba a la conclusión que si debido a la suerte $\mathrm{X}$ nace en una cultura cuyo acceso no es gratuito, ya que lo costoso de su decisión no se debe a sus elecciones, entonces debe ser compensado. Como se puede ver, este esquema justificatorio de distribución de recursos se construye sobre la noción de un individuo, y las condiciones que hacen que consideremos que debe o no hacerse responsable de su elección. En el fondo, parte de un concepto de individuo autónomo como el criterio con el que debemos tomar decisiones distributivas, negando la compensación cuando la

\footnotetext{
${ }^{2}$ Un enfoque parecido es desarrollado por G.A. Cohen (2004).

252 | AlPHA № 52 (Julio 2021) PÁGS. 243-260. ISSN 07 16-4254
} 
decisión responde de alguna manera a este ideal de autonomía y aceptándola cuando no es así.

El problema de este enfoque igualitarista de la suerte es que para evaluar medidas compensatorias para los pueblos indígenas quizá este enfoque no es apropiado. Ciertamente parece prudente dejar la puerta abierta para considerar que un enfoque corregido del igualitarismo de la suerte, o este en conjunto a otro enfoque de la justicia, sean apropiados para responder a los reclamos de los pueblos indígenas, pero así como Kymlicka elabora su argumento este es sobre todo problemático. El problema es que el igualitarismo parece considerar la parte por el todo, porque interpreta las situaciones de injusticia como un asunto de distribución de recursos que afecta al bienestar (nótese) de los individuos. Para el igualitarismo de la suerte es suficiente establecer que la causa de la elección costosa es consecuencia de la suerte y no de la decisión de una persona para establecer que la compensación es debida. Sin embargo, los únicos aspectos que entran en la consideración de lo que constituye "decisión" y lo que constituye "suerte" son hechos relativos a la posibilidad de una elección individual que responde al ideal de autonomía. Es decir, identificar porque una persona no es responsable de sus elecciones costosas no requiere identificar la posibilidad que dicho costo tenga su explicación en hechos de injusticia históricos ${ }^{3}$. Estoy aquí considerando la poco controversial afirmación presente en los debates referentes a la justicia global, que el colonialismo es la causa fundamental que explica la pobreza de los países del tercer mundo ${ }^{4}$. Sin embargo, el colonialismo también explica la causa del acceso costoso de los pueblos indígenas a sus propias culturas: la pérdida de la autodeterminación y la sujeción no consentida a la autoridad de los Estados europeos o de sus descendientes, como ocurre en Chile y en las Américas.

Ahora bien, si en la identificación de las causas o factores considerados para establecer una obligación compensatoria por parte del Estado hacia los pueblos indígenas no figuran las injusticias históricas, ¿cómo explicamos la existencia de numerosas comisiones en distintos países que han tenido por finalidad reconstruir hechos de injusticias históricas, identificar sujetos colectivos responsables, y sujetos colectivos que han sufrido el costo de la colonización, como la "Comisión Verdad Histórica y Nuevo Trato con los Pueblos Indígenas" en Chile? La importancia política del reconocimiento de las injusticias históricas y su potencial para reestructurar las relaciones entre los

\footnotetext{
${ }^{3}$ Es posible que esta incapacidad del igualitarismo para considerar las injusticias históricas se explique en que el igualitarismo de la suerte no es una teoría moral "completa". Como afirma Lippert-Rassmusen, el igualitarismo de la suerte no tiene mucho que decir acerca de la permisibilidad de los comportamientos (Lippert-Rassmusen, 2016, pp. 2-3) cuando estos no generan consecuencias distributivas moralmente relevantes. Aparece, eso sí, porque el igualitarismo de la suerte puede ser compatible con otras comprensiones de la injusticia, que parece prudente no rechazar esta doctrina de manera concluyente.

${ }^{4}$ Para una panorámica general pertinente a las causas de la pobreza global y las posibles respuestas, véase Pogge (2008).
} 
pueblos indígenas y la sociedad chilena, parece suficiente motivo para corregir o abandonar la teoría igualitarista de la suerte. Sin embargo, no solo hay razones políticas que abonan dicha corrección o abandono, sino de justicia y legitimidad de la acción estatal. Si no somos capaces de reconocer el derecho de los pueblos indígenas a acceder a su cultura, es decir, a una cultura determinada, todo lo que queda es un derecho amplio a acceder a un contexto cultural indeterminado. En otras palabras, si no somos capaces de conectar la pérdida histórica de su cultura particular con los reclamos presentes de acceder a su cultura, entonces sus reclamos de acceder a "su cultura" parecen menos plausibles, al menos entendido como un reclamo substantivo a acceder no solo a sus prácticas culturales, sino a una situación de autodeterminación política. Si no hay conexión, todo lo que queda es un derecho al acceso indiferenciado a un contexto cultural, cualquiera que sea este, no muy diferente del derecho del tipo (2) que eventualmente podría beneficiar a los grupos étnicos.

\section{EL DERECHO A LA CULTURA Y LAS INJUSTICIAS HISTÓRICAS}

Considero que el reclamo sustantivo de los pueblos indígenas no está de manera directa referido al acceso a $\mathrm{CU}_{2}$, sino que este se deriva de sus reclamos más fundamentales a la autodeterminación política. Esta comprensión sustantiva del acceso a $\mathrm{CU}_{2}$ supone reinterpretar los reclamos del acceso a la cultura como reclamos a la pérdida de la autodeterminación, esto es, no como teniendo solo un carácter "cultural", sino de carácter sobre todo político. Esta forma alternativa de entender la pérdida de la cultura y que conecta con los reclamos de la autodeterminación, supone demostrar que existe una conexión estrecha entre el control de los mecanismos de reproducción política de un pueblo y los mecanismos de reproducción cultural ${ }^{5}$. Centrándose en la pérdida de la autodeterminación de los pueblos indígenas, el argumento asume que existe una conexión entre la autodeterminación y el acceso a la cultural societal tal que el acceso a la cultura societal está implicado en el hecho que un pueblo sea autodeterminado. Esta última comprensión de la pérdida de la cultura es, desde luego, indirecta, porque en realidad está dirigido a establecer la pérdida de la cultura que se sigue de la pérdida de la autodeterminación. Debo advertir que el desarrollo de esta perspectiva que denomino "multiculturalismo crítico" no será elaborado en este ensayo, pero es este argumento el que de manera precisa establece las bases fundamentales para su elaboración.

Ya hemos trazado un camino para elaborar la crítica al multiculturalismo liberal y que de forma simultánea me permitirá identificar el fundamento central para justificar el acceso a la cultura societal de los pueblos indígenas. Este camino requiere explorar más de cerca la conexión entre la pérdida histórica de $\mathrm{CU}_{2}$ de los pueblos indígenas, entendida

\footnotetext{
${ }^{5}$ Un argumento similar ha sido desarrollado por Patten (2014, pp. 232-256; 2018, p. 134).

254 | ALPHA № 52 (Julio 2021) PÁGS. 243-260. ISSN 07 16-4254
} 
como la pérdida de un contexto en el que el acceso era gratuito ${ }^{6}$. Para ello consideraré la controversia entre Will Kymlicka y Jeremy Waldron respecto del derecho a la cultura, porque demuestra sobre todo que, si no podemos establecer la mencionada conexión, no es posible sostener la existencia de un reclamo sustantivo a acceder a $\mathrm{CU}_{2}$. Es otras palabras, el debate es crucial para mi argumento porque demuestra que, si no existe conexión, lo que quedaría sería propiamente un derecho o facultad dirigida a acceder a ciertos fragmentos de una cultura, cualquier sea esta. Es sobre todo esta la postura implícita de Kymlicka, aunque para llegar a esta conclusión, que se sigue de la premisa liberal de la justicia multicultural, debo elaborar el argumento. La controversia entre Kymlicka y Waldron es sobre todo una controversia por el alcance del derecho a la cultura. Kymlicka, por un lado, pareciera sostener una posición que considera que las minorías deberían poder acceder a "su cultura", mientras que Waldron sostiene que es suficiente para el trato igualitario que las minorías puedan acceder a "una cultura", cualquiera sea esta. Lo siguiente describe a grandes rasgos el argumento de Waldron:

Desde luego, la elección tiene lugar en un contexto cultural, entre opciones que tienen significados culturalmente definidos. Pero al desarrollar su argumento, Kymlicka es culpable de algo así como la falacia de la composición. Del hecho que cada opción tenga un significado cultural, no se sigue que deba existir una única perspectiva en la cual cada opción disponible tenga asignada un significado. Las opciones significativas pueden venir como ítems o fragmentos de una variedad de orígenes culturales (Waldron, 1992, p. 783) ${ }^{7}$.

El argumento de Waldron está basado en una interpretación cosmopolita del acceso a la cultura. Waldron sostiene que no es necesario que las opciones de las personas deban ser significativas en un contexto determinado, porque según él, todavía podemos elegir de manera significativa cuando los contextos culturales varían, siempre que las opciones que tengamos disponibles sean razonables. En otras palabras, tanto Waldron como Kymlicka sostienen que los contextos culturales son parte de la estructura básica de la sociedad, pero a diferencia de Kymlicka, Waldron limita el alcance del derecho a la cultura. Para Waldron el derecho que es parte de la estructura básica de la sociedad, y que se encuentra detrás del velo de la ignorancia, no va referido a una cultura en particular. No existe algo así como el derecho a acceder a una cultura societal determinada, sino el derecho a alguna $\mathrm{CU}_{2}$. En otras palabras, no existe un derecho a acceder a una cultura

\footnotetext{
${ }^{6}$ He elegido esta comprensión de la pérdida de la cultura porque hace referencia a la pérdida de $\mathrm{CU}_{2}$, porque deja abierta la posibilidad de corregir los problemas que presenta el igualitarismo de la suerte mediante una comprensión más amplia, y porque deja abierta la posibilidad de establecer una conexión entre la pérdida de la cultura y la pérdida de la autodeterminación tal que sea posible desarrollar una interpretación "sustantiva" del reclamo a acceder a $\mathrm{CU}_{2}$.

${ }^{7}$ Con posterioridad Waldron ha moderado, aunque no abandonado, sus posturas al respecto Waldron (2002). 
determinada. Las minorías no tienen derecho a su cultura, sino a una cultura ${ }^{8}$, cualquiera que esta sea. Kymlicka responde a Waldron afirmando que aun cuando sea cierto que las opciones que los individuos tienen para elegir provengan de varias fuentes culturales, lo que hace que esta pluralidad de fuentes esté disponible es que, en primer lugar, estén disponibles. Es decir, para tener una variedad de contextos para elegir, los individuos necesitan que existan diferentes culturas, y esta es sobre todo la incertidumbre que experimentan las minorías culturales. Esta disponibilidad de contextos culturales es asumida por Waldron, pero esto sería lo que de acuerdo con Kymlicka no puede asumirse cuando se trata de derechos de acceso a $\mathrm{CU}_{2}$. Sin derechos para acceder a una cultura particular y que aseguren de alguna manera que las diferentes culturas se mantengan en el tiempo, no podría existir la diversidad de contextos culturales que las personas pueden elegir, y por tanto el argumento de Waldron no invalidaría la perspectiva de Kymlicka. En definitiva, parece afirmar Kymlicka, si no hay variedad de contextos culturales para elegir, la elección no puede ser razonable.

El problema es que la tesis de Kymlicka, al final de cuentas, es muy similar a la de Waldron. Waldron sostiene una tesis sobre los derechos especiales muy débil describible como una obligación negativa del Estado en términos de un deber de no interferencia, similar al deber de no interferencia en materia de religión (Kymlicka, 1995, p. 85), pero la tesis de Kymlicka, a pesar suyo, no llega mucho más lejos que la de Waldron. En efecto, sí debe reconocerse que Kymlicka va más lejos que Waldron en el sentido que considera insuficiente para proteger los derechos de las minorías la existencia para el Estado de un deber negativo de no interferencia. Para Kymlicka, los tres tipos de derechos especiales incluyen obligaciones negativas, pero son sobre todo derechos positivos. Por esta razón, Kymlicka es más exigente que Waldron en las condiciones que el Estado debe cumplir para afirmar que ha otorgado un trato igualitario a las minorías. Sin embargo, para Kymlicka los derechos especiales tienen límites semejantes, semejantes si atendemos a los fundamentos que justifican los derechos especiales. Ambos, Waldron como Kymlicka, justifican la existencia de ciertos derechos especiales para las minorías, en atención al valor central de sus concepciones del liberalismo: la autonomía individual. Como resultado, ambos recurren a las mismas consideraciones, aunque sujetas a exigencias en efecto diferentes, para limitar las obligaciones del Estado en materia de derechos especiales.

Como hemos visto en la sección 2, Kymlicka considera que no están justificados los derechos especiales cuando producen como consecuencias o pueden ser interpretados como restricciones internas. La razón de estas restricciones es evitar que el Estado beneficie prácticas culturales que restrinjan o violen derechos liberales, es decir, prácticas

\footnotetext{
${ }^{8}$ El debate en inglés es entre quienes entiende el derecho "to the culture" versos quienes entienden que es un derecho "to $a$ culture". Espero haber capturado con mi traducción la razón de la diferencia entre ambas acepciones. 256 | AlPHA № 52 (JuLIO 2021) PÁGS. 243-260. ISSN 07 16-4254
} 
que no promuevan o respeten la autonomía individual. Se sigue que el diseño de la teoría de Kymlicka es aquí del todo consistente con el valor central que le da valor (liberal) a la práctica, la autonomía de la persona. Sin embargo, Kymlicka parece no notar que este enfoque no solo no justifica obligaciones negativas para el Estado, sino que justifica la interferencia de los grupos minoritarios. La protección de los derechos liberales, ya sea que tenga lugar mediante mecanismos administrativos, policiales o legales como el derecho penal", establece obligaciones "positivas" del Estado para evitar su violación. Solo considerando que existen estas obligaciones positivas que facultan la interferencia de los grupos minoritarios, reduciendo de manera significativa el alcance de las protecciones externas, es que puede entenderse lo que Kymlicka afirma respecto de cómo debe proceder el Estado cuando residen en su interior grupos no liberales, ya sea minorías nacionales o grupos étnicos.

Kymlicka afirma, como respuesta a Waldron y otros liberales que no ven de forma positiva a los grupos minoritarios como grupos que pueden contribuir al bienestar de las personas, respecto de las culturas no liberales, la tarea del Estado liberal no debe ser suprimirlos. Para Kymlicka, el Estado liberal debe tener un objetivo diferente, el Estado liberal debe tener por finalidad "liberalizarlos". En palabras de Kymlicka, el objetivo:

no debería ser disolver las naciones no liberales, sino que buscar liberalizarlas. Esto puede no ser siempre posible. Pero vale la pena recordar que todas las naciones liberales existentes tenían pasados no liberales, y que su liberalización requirió un proceso prolongado de reforma institucional. Asumir que cualquier cultura es inherentemente iliberal, e incapaz de reformarse, es etnocéntrico y ahistórico (Kymlicka,1995, p. 94).

La postura de Kymlicka, sería más benevolente que las posturas con quienes hace la comparación, es entender facultado al Estado liberal para intervenir en los asuntos internos de las minorías que existen dentro de su territorio. Es decir, existen obligaciones positivas como negativas para promover y proteger los derechos y prerrogativas liberales, de modo de promover y proteger la autonomía individual. Esta perspectiva adopta entonces una comprensión que podríamos calificar como "postcolonial" (Young, 2016, p. 44, trad. del autor), sobre el acceso de las minorías a $\mathrm{CU}_{2}$. En términos de disponibilidad de contextos culturales, la posición de Kymlicka se acerca mucho a la propuesta de Waldron de que lo que él está dispuesto a reconocer. En efecto, si se tratara de aceptar un reclamo sustantivo de acceso a la cultura societal, entonces no podría justificarse la "liberalización" de las culturas iliberales, porque las minorías tendrían un derecho de acceso a "su cultura". Por el contrario, si el acceso es limitado entonces el acceso que

\footnotetext{
${ }^{9}$ Si bien Kymlicka no defiende de manera explícita el uso del derecho penal y el castigo en general para prevenir prácticas que afectan de forma grave derechos básicos, Kymlicka sí acusa de "conservadurismo" a los líderes indígenas que defienden este tipo de prácticas, y celebra el enfoque de gobernanza indígena basado en derechos humanos liberales, véase Kymlicka (2007, pp. 149-151).
} 
existe, o que en todo caso está el Estado chileno dispuesto a ofrecer, es un derecho a "la cultura", incluyendo ciertos fragmentos tolerables por la justicia política liberal. Como hemos visto, esta es la forma de comprender la estrategia de Kymlicka de limitar los derechos especiales a las protecciones externas. No es otra la estrategia que hasta hoy ha sido empleada por los diferentes gobiernos chilenos.

\section{CONCLUSIÓN, HACIA UN MULTICULTURALISMO CRÍTICO}

La conclusión a la que podemos llegar, luego de este examen de los fundamentos de la teoría de Kymlicka, es que tan bien esta logra ilustrar y hacer manifiesto los compromisos teóricos, las limitaciones del multiculturalismo liberal y el diseño de la política de derechos especiales. Las diferentes políticas adoptadas por el modelo filosófico liberal, en efecto, representan un avance en comparación con las diversas políticas previas, como la era de exterminio y posterior era asimilacionista de los pueblos indígenas. Asimismo, el multiculturalismo liberal tiene el mérito de haber posibilitado un mayor debate público acerca de los derechos culturales y ha contribuido, de forma tímida, y quizás de manera indirecta, a la movilización política de los pueblos indígenas. Este incremento de la movilización política se debe sobre todo a la participación activa de los propios miembros de los pueblos indígenas en movimientos locales e internacionales por la lucha de un acceso igualitario a sus $\mathrm{CU}_{2}$. Sin embargo, esta perspectiva teórica parece haberse estancado, y ello, creo, se debe fundamentalmente al carácter despolitizado que está implícito en la estructura de acomodación y reconocimiento basado en derechos individuales. Lo que hace necesario, desde un punto de vista filosófico, fortalecer y promover un acercamiento político a los derechos culturales, el que debería ser el objetivo principal del multiculturalismo crítico.

Es posible concluir entonces que el multiculturalismo crítico no debe restringirse a considerar aceptables reclamos meramente negativos como el de Waldron, o positivos como el de Kymlicka, sino ampliarse y centrarse en reclamos sustantivos de acceso a $\mathrm{CU}_{2}$ de los pueblos indígenas. En particular, es necesario una perspectiva teórica que nos permita conectar las injusticias históricas con los reclamos presentes de los pueblos indígenas para acceder a su cultura societal, y haga justicia a la posición que los pueblos indígenas merecen como un sujeto político de carácter colectivo y autodeterminado. Esta nueva perspectiva teórica, que he denominado multiculturalismo crítico, no solo debería ofrecer una vía para conectar las injusticias históricas con los reclamos para acceder a una cultura societal particular, sino que además debe permitir y promover la politización de la justicia multicultural. Ciertamente, los reclamos acerca de la autodeterminación no 
pueden entenderse solo como reclamos basados en derechos individuales ${ }^{10}$, porque se trata de reclamos colectivos para el ejercicio del poder político en un territorio determinado, y este tipo de reclamos, no pueden sino ser de carácter colectivo y político.

\section{OBRAS CITADAS}

Arneson, Richard (2013). "Egalitarianism", The Stanford Encyclopedia of Philosophy, Summer 2013 Edition, consultado 27 de enero 2019, disponible en: https://plato.stanford.edu/entries/egalitarianism/

Atria, Fernando (2004a). “Existen derechos sociales?”, Discusiones 4: 25-59.

(2004b). "Derecho y política a propósito de los derechos sociales", Discusiones 4: 145-176.

Bouchard, Gerard (2011). "What is interculturalism", McGill Law Journal 56.2: 435-468.

Carens, Joseph (2000). Culture, citizenship, and community: a contextual exploration of justice as evenhandedness. Oxford: Oxford University Press.

Cohen G.A. (2004). "Expensive taste rides again", en Justine Burley (ed.) Dworkin and his critics: with replies by dworkin. Chichester: Blackwell Publishing: 3-29.

Dworkin, Ronald (2013). "What is equality? Part 2: equality of resources", Philosophy and public affairs 10.4: 283-345.

Hirose, Iwao (2015). Egalitarianism. Abingdon: Routledge.

Knight, Carl (2013). “Luck egalitarianism”, Philosophy compass 8.10: 924-934.

Kymlicka, Will (2016). "Defending diversity in an era of populism: Multiculturalism and interculturalism compared", en Nasar Meer, Tariq Modood, y Ricard ZapataBarrero (eds.) Multiculturalism and interculturalism: debating the dividing lines. Edinburgh: Edinburgh University Press: 158-177.

- (2007). Multicultural odysseys: navigating the new international politics of diversity. Oxford: Oxford University Press.

_ (1995). Multicultural Citizenship. Oxford and New York: Clarendon Press.

Lippert-Rasmussen, Kasper (2016). Luck egalitarianism. London and New York: Bloomsbury.

_ (2011). "Immigrants, multiculturalism, and expensive cultural tastes: Quong on luck egalitarianism and cultural minority rights", Les ateliers de l'éthique / The Ethics Forum 6.2: 176-192.

Miller, David (2006). "Political philosophy", en Edward Craig (ed.) Routledge Encyclopedia of Philosophy. London: Routledge: 815-818.

\footnotetext{
${ }^{10}$ En general sobre la estructura de los derechos véase Rainbolt (2007). En la medida que los derechos culturales son comprendidos como derechos sociales, se hacen acreedores de la crítica de Atria relativa al efecto individualizador de los derechos, véase Atria (2004a), Atria (2004b).
} 
Modood, Tarik (2017). "Must interculturalists misrepresent multiculturalism?", Comparative Migration Studies 5.15: 1-17. No CITADO

Parekh, Bhikhu (2000). Rethinking multiculturalism: Cultural diversity and political theory. London: MacMillan Press.

Patten, Alan (2018). "Liberalism, culture and recognition: A reply to critics", Political Theory 46.1: 131-141.

_ (2014). Equal Recognition: The moral foundations of minority rights. Princeton: Princeton University Press.

Pogge, Thomas (2008). World poverty and Human Rights: Cosmopolitan responsibilities and Reforms, Second edition. Cambridge: Polity Press.

Rainbolt, George (2007). "Rights theory", Philosophy compass 1.1: 11-21.

Taylor, Charles (2012). "Interculturalism or multiculturalism?", Philosophy and social criticism 38.5: 413-423.

Waldron, Jeremy (2002). "One law for all? The logic of cultural accommodation", Washington and Lee Law Review 59: 3-34.

_ (1992). "Minority cultures and the cosmopolitan alternative", Michigan Journal of Law Reform 25.3: 751-793.

Young, Robert (2016). Postcolonialism: An historical introduction. Chichester: Wiley Blackwell. 